\title{
Cine con valores y docencia universitaria
}

\section{Susana Collado-Vázquez ${ }^{a}$, Jesús María Carrillob}

aDepartamento de Fisioterapia, Terapia Ocupacional, Rehabilitación y Medicina Física. Facultad de Ciencias de la Salud. Universidad Rey Juan Carlos. susana.collado@urjc.es bepartamento de Personalidad, evaluación y Psicología clínica. Facultad de Psicología de la Universidad Complutense de Madrid.

\begin{abstract}
During the months of February and March 2015, the fourth course of Cinema with values was celebrates for students of the King Juan Carlos University. This course was held within the program of Human Rights of that University.

The course objective was to stimulate reflection on human values using film as a tool.

Three sessions in which film fragments were projected were made, a cinemaforum was held face and virtual blog CINEMED, and attendees worked in small groups, they participated in discussions and delivered a paper on values and cinema. The methodology used skills such as teamwork, reflection, critical analysis and oral and written communication is encouraged.

After completing the course the students responded anonymously and voluntarily the ad hoc questionnaire, which showed a high degree of satisfaction with the methodology and course content.
\end{abstract}

Keywords: Cinema; Teaching; Human values; Transversal skills; Human Rights 


\begin{abstract}
Resumen
Durante los meses de febrero y marzo de 2015 se celebró el IV Curso de Cine con Valores dirigido a estudiantes de la Universidad Rey Juan Carlos. Este curso se realizó dentro del programa de Derechos Humanos de la citada Universidad.

El objetivo del curso fue estimular la reflexión sobre valores humanos utilizando el cine como herramienta.

Se realizaron tres sesiones en las que se proyectaron fragmentos fílmicos, se realizó un cine-fórum presencial y otro virtual en el blog CINEMED, y los asistentes trabajaron en pequeños grupos, participaron en debates y entregaron un trabajo sobre valores y cine. Con la metodología utilizada se fomentaron competencias como el trabajo en equipo, la reflexión, el análisis crítico y la comunicación oral y escrita.

Al finalizar el curso los estudiantes contestaron de forma anónima y voluntaria el cuestionario elaborado ad hoc, en el que manifestaron un alto grado de satisfacción con la metodología y con los contenidos del curso.
\end{abstract}

Palabras clave: Cine; Docencia; Valores; Competencias transversales; Derechos humanos.

\title{
Introducción
}

El cine se ha utilizado en numerosas ocasiones como herramienta docente en los diversos niveles educativos con diversos objetivos y diferente metodología, obteniéndose muy buenos resultados y resultando ser actividades atractivas y amenas para los estudiantes. Mediante la utilización del séptimo arte pueden ilustrarse diversos temas y ayudar a los estudiantes a la adquisición de competencias transversales (1).

El cine cuenta muchas historias de diversas épocas y con contenidos y personajes de todo tipo y existen muchos títulos de utilidad para analizar los valores o la falta de los mismos y ayudar a reflexionar sobre diversos aspectos. $\mathrm{Y}$ asimismo poder poner en relación problemas que aparecen en los filmes con situaciones realies de la vida cotidiana. Existen numerosas publicaciones, tanto artículos como libros en los que se recoge la relación entre el cine y los valores humanos y la ética, y algunas aplicaciones docentes, que da una idea de 
la importancia de este tema y de las posibilidades que el séptimo arte tiene como herramienta docente y divulgativa (1-5).

Por ello, por cuarto año consecutivo pusimos en marcha el curso de cine con valores para fomentar el análisis de los valores humanos y estimular competencias transversales en los estudiantes.

\section{Objetivos}

Los objetivos fueron estimular la reflexión sobre valores humanos utilizando el cine como herramienta didáctica y facilitar la adquisición de competencias transversales, tales como el trabajo autónomo y colaborativo, la capacidad de observación, la reflexión, el análisis crítico y la comunicación oral y escrita.

\section{Desarrollo de la innovación}

Por cuarto año consecutivo se desarrolló en la Facultad de Ciencias de la Salud de la Universidad Rey Juan Carlos (Alcorcón, Madrid) el Curso de Cine con Valores, abierto a todos los alumnos de la Universidad. Durante este curso 2014-2015 el curso se ha desarrollado dentro del Programa de Derechos Humanos de la Universidad Rey Juan Carlos. La duración fue de 25 horas.

Se dio publicidad al curso a través del blog de la URJC, mediante el blog CINEMED, a través del blog de Derechos Humanos de la URJC, en redes sociales (Twitter) y a través de los tutores integrales de diversas titulaciones.

Se llevaron a cabo tres sesiones en las que se proyectaron fragmentos fílmicos relacionados con las temáticas seleccionadas para la cuarta edición del curso: discapacidad, dignidad humana y responsabilidad social. Los asistentes recibieron material complementario relacionado con los temas tratados (artículos, títulos de películas, cortos y documentales, fichas, cuestionarios, lecturas recomendadas, temas para reflexionar).

El primer día se realizó una introducción y justificación del curso sobre los valores que se muestran en el cine y empleando diversos ejemplos y fragmentos fílmicos para ilustrar la relación entre el séptimo arte y la transmisión de valores. Algunos de los títulos utilizados para tal fin fueron: Capitanes intrépidos, La vida es bella, Qué bello es vivir, Caballero sin espada, El club de los poetas muertos, El doctor, Patch Adams, Marcelino Pan y vino, El árbol del ahorcado, Cadena de favores, entre otros títulos. A continuación se abordó la discapacidad desde diversos puntos de vista, analizando diversas situaciones en las que se vulneran los derechos de las personas con diversidad funcional, y ofreciendo una visión de la imagen que el cine ha dado de la discapacidad. Para esta segunda parte de la sesión se emplearon también numerosos fragmentos fílmicos de títulos como: Las llaves de casa, Hombres, El milagro de Ana Sullivan, Forrest Gump, Yo soy Sam, Freaks, Iris, Lejos de ella, Arrugas, My name is Lisa, Algo queda, o Un mundo a su medida.

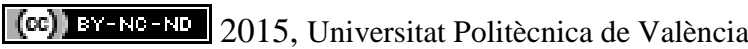


Al finalizar las exposiciones se formaron pequeños grupos de 4-5 estudiantes para reflexionar sobre las cuestiones planteadas. Se les facilitó material complementario, artículos y un listado de preguntas para orientar el trabajo en equipo. Una vez realizado el trabajo en grupo se desarroló un debate en el que participaron todos los grupos.

En la segunda sesión se abordaron dos temas: la dignidad humana y la responsabilidad social. Para ilustrar el primero de los temas seleccionados se emplearon fragmentos fílmicos de películas tales como El hombre elefante, agua, Camino a Guantánamo, Mi pie izquierdo, Río Bravo, 12 años de esclavitud, Dumbo, entre otras.

En relación con la responsabilidad social se emplearon fragmentos de cortos, documentales y filmes comerciales como Super size me, Sicko, Wall-e, Los juegos del hambre o Erin Brockovich por citar algunos ejemplos.

Como en la primera sesión los asistentes formaron pequeños grupos y trabajaron con el material complementario que se les entregó. Para finalizar la sesión tuvimos tiempo para desarrollar un debate.

La tercera sesión consistió en un cine-fórum presencial utilizando la película La princesa prometida. A cada alumno se le entregó información sobre el filme: fichas técnica y artística, sinopsis, anécdotas del filme y cuestiones para el debate, por ejemplo, qué era lo que más y menos les había gustado, descripción de los personajes en relación con los valores y qué valores se defendían o vulneraban en este filme.

El cine-fórum comenzó con la presentación de la película, con información sobre la misma, la época en la que se realizó, datos sobre la dirección, guión, reparto, música y diversas anécdotas sobre el filme. A continuación se procedió a la proyección del título seleccionado y tras visionar el filme se formaron pequeños grupos que comentaron aspectos relacionados con los valores de la película y respondieron a las cuestiones planteadas. Finalizó la sesión con un debate para poner en común las reflexiones y opiniones de todos los pequeños grupos.

Además de las actividades presenciales se realizó un cine-fórum virtual en el blog CINEMED sobre la película Mi pie izquierdo (http://suscinemed.blogspot.com.es/2015/02/cine-forum-mi-pie-izquierdo-iv-curso-de.html), y se pidió a los alumnos la realización de un trabajo individual en el que tenían que contestar a unas preguntas y comentar acerca de una película de su elección en la que estuvieran presentes ciertos valores (FIGURA 1)

Por último los asistentes respondieron a un cuestionario de satisfacción que se elaboró específicamente para este curso de Cine con valores. 


\section{Resultados}

El curso se ofertó a todos los estudiantes de la Universidad Rey Juan Carlos y se cubrieron las 50 plazas del mismo. Los alumnos procedieron, fundamentalmente, del grado de Fisioterapia, pero también de otras titulaciones como los Grados de Educación Infantil, Terapia Ocupacional, Medicina, Comunicación Audiovisual e Ingeniería Informática.

\section{Cinemed}

En este blog encontrarás información y podrás opinar sobre Cine y Medicina y sobre la aplicación del séptimo arte en la docencia de ciencias de la Salud.

jueves, 12 de febrero de 2015

Cine fórum: Mi pie izquierdo. IV Curso de Cine Con valores

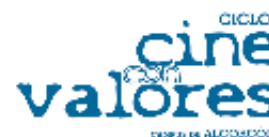

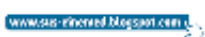

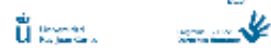

En el IV Curso de cine con valores celebrado en 2015 dentro del Programa de Derechos Humanos de la Universidad Rey Juan Carlos, vamos a dedicar la actividad de cine-Fórum virtual a la película mi pie izquierdo (Jim sheridan, 1989).

ü̉ argumento en el siguiente enlace:

Wi pie izquierdo. Filmaffinity

A continuación os dejo un vídeo en el que podréis ver un trailer de esta película hacada en hecho reales en concreto en el relato autohiográfico de christy

Los asistentes mostraron una actitud muy participativa, se generó mucho debate y los alumnos se mostraron satisfechos con el trabajo en equipo.

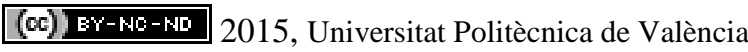

Congreso In-Red (2015)
Buscar este blog

Buscar

Introduce tu dirección de

correo y siguenos por

email.verificad en el correo

de confirmación

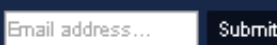

cine y ciencias de la salud.

Aplicaciones docenter

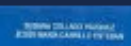


En el cuestionario de valoración los alumnos puntuaron muy favorablemente el curso, al profesorado, los contenidos, los temas tratados y la metodología de trabajo. Entre los comentarios la mayoría volvería a realizar un curso de similares características y lo recomendarían a otros compañeros, y afirmaron también que habían aprendido de forma dinámica y amena, que les hubiera gustado que el curso estuviera compuesto de más sesiones para tratar otros temas y valores. Como sugerencias propusieron que en otras ocasiones se hablara de bioética, de la libertad, la justicia, la violencia, las adicciones, entre otras temáticas.

También nos indicaron que querían que se proyectaran películas y fragmentos fílmicos de títulos más recientes, o que se complementaran con fragmentos de series televisivas, o más referencias literarias y artísticas.

Preguntados acerca de la adquisición de competencias los alumnos indicaron que el curso les había ayudado a reflexionar sobre los valores, a tener en cuenta las opiniones de otros compañeros de diversas titulaciones, que la dinámica utilizada de trabajo individual, en equipo, debates, les había enriquecido, y que les gustaría que estas metodologías se emplearan en otros cursos.

\section{Conclusiones}

La utilización del cine para fomentar la reflexión sobre valores humanos se muestra como una valiosa herramienta didáctica muy bien valorada por los alumnos.

Los estudiantes manifiestan que el curso les ha sido de utilidad para fomentar la adquisición de diversas competencias transversales, tales como la capacidad de observación y reflexión crítica, el trabajo autónomo y colaborativo y la comunicación oral y escrita.

Los alumnos se han mostrado satisfechos con la actividad y han manifestado su interés en participar en otros cursos similares y han aportado algunas sugerencias de interés para la mejora del curso.

\section{Referencias}

1. Collado-Vázquez S, Carrillo JM. Cine y Ciencias d ela sxlaud. Aplicaciones docentes. Madrid: Dykisnon;2013.

2. Equipo padres y maestros. Cine y ciudadanía. Valores para trabajar en el aula. Bilbao:Mensajero;2009.

3. Tomás y Garrido MC, Ródenas Tolosa B. Los sentimientos y la vida afectiva a través del cine. Madrid: Ediciones Internacionales Universitarias; 2009

4. Soto Nieto F, Fernández FJ. Entre Dios y los hombres. La práctica médica y científica a través del cine. Madrid: Dykinson;2010

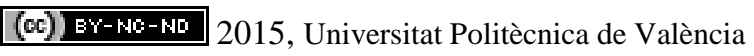


5. García Pelegrín JM. El cielo sobre Hollywood. Madrid:Palabra;2009.

\section{(cc) EY-NC-ND 2015, Universitat Politècnica de València}

